\title{
RF-current effects on intrinsic Josephson junctions
}

\author{
S. Saleem, J.C. Fenton, M. Korsah, S. Speller, C.R.M. Grovenor, and P.A. Warburton
}

(C) 2009 IEEE. Personal use of this material is permitted. Permission from IEEE must be obtained for all other uses, in any current or future media, including reprinting/republishing this material for advertising or promotional purposes, creating new collective works, for resale or redistribution to servers or lists, or reuse of any copyrighted component of this work in other works.

\begin{abstract}
We have fabricated intrinsic Josephson junction arrays using $\mathrm{Tl}_{2} \mathrm{Ba}_{2} \mathrm{CaCu}_{2} \mathrm{O}_{8}$ thin films. The junctions have dimensions of order $1 \mu \mathrm{m}^{2}$ and therefore high slope resistance in the range $1 \mathrm{k} \Omega$ to $10 \mathrm{k} \Omega$ per junction on the quasiparticle branches. We have irradiated the junctions with microwaves at a frequency of $6.37 \mathrm{GHz}$. We observe both microwave-induced vortex-flow branches and strong suppression of the switching current but only when no junctions are in the voltage state. We attribute this observation to the shunting of the high-resistance junctions by the environmental impedance, and discuss the significance of this result for the application of intrinsic junctions as THz sources and qubits.
\end{abstract}

Index Terms - Josephson arrays, Josephson oscillators, Josephson vortex flow

\section{INTRODUCTION}

$I^{2}$

NTRINSIC Josephson junctions are formed by alternating layers of superconducting and insulating material within the unit-cell of a cuprate high temperature superconductor [1]. Intrinsic Josephson junction (IJJ) arrays are tuneable oscillators with narrow linewidth in the THz range [2]. By coherently coupling many IJJs together, all of which are in the voltage state, it may be possible to achieve power output levels and linewidths in the THz regime which are competitive with those currently achievable using rival technologies such as the quantum cascade laser [3]. In addition IJJs, by virtue of the fact that they contain no artificially-engineered barrier, may possibly be suitable for use as Josephson qubits with potentially long decoherence lifetimes [4]. Manipulation of such qubits is done by pulsed microwave irradiation. It is therefore important to understand the electrodynamics of coupled IJJs and how this depends upon the number of junctions which are in the voltage state.

We have therefore measured the variation of the static current-voltage characteristics of an array of IJJs when it is irradiated at microwave frequencies. Specifically we study the dependence of the switching current upon the microwave field and how this depends upon the number of junctions in the array which are in the voltage state. In addition we have observed microwaveinduced vortex-flow branches, but it is notable that these cannot be observed when one or more junctions is in the voltage state.

\section{EXPERIMENTAL DETAILS}

Device fabrication starts by growing a $\mathrm{Tl}_{2} \mathrm{Ba}_{2} \mathrm{CaCu}_{2} \mathrm{O}_{8}$ (TBCCO) film of nominal thickness $500 \mathrm{~nm}$ on a $10 x 10 \mathrm{~mm}\left[\begin{array}{lll}0 & 0 & 1\end{array}\right]-$ oriented $\mathrm{LaAlO}_{3}$ substrate. A gold layer about 200nm in thickness is deposited ex-situ on top to protect the superconducting film during processing. Full details of film growth and structural characterization have been previously reported [5].

The device pattern is transferred onto the thin film using conventional optical lithography. The masked films are then milled by a $1 \mathrm{kV}$ argon ion-beam to create tracks of width $4 \mu \mathrm{m}$. The current-voltage characteristics of each track are measured at this stage at $4.2 \mathrm{~K}$ to ensure that there are no junctions in the c-axis films arising from grain boundaries or misaligned a-axis oriented film regions.

IJJ arrays are then created using a Carl Zeiss XB1540 $30 \mathrm{kV}$ gallium focused-ion-beam (FIB) equipped with an in-situ scanning electron microscope (SEM). We follow the technique first reported in [6]. With the ion beam oriented perpendicular to the substrate surface, the track is narrowed down from both sides to a width of one micron using 100pA ion beam current followed by a $10 \mathrm{pA}$ "polish" of the side walls of the track. The sample is then reoriented so that the ion beam is almost parallel to the substrate - typically it makes an angle of $5^{\circ}$ to the substrate surface. Two lateral slots are drilled in the track with a $10 \mathrm{pA}$

This work is supported by EPSRC, UK, grant reference EP/D029783/1.

S. Saleem, J.C. Fenton and P.A. Warburton are with University College London, London Centre for Nanotechnology, 17-19 Gordon Street, WC1H 0AH, London, UK (phone: +44 (0)20 76790639 ; fax +44 (0)20 76790595 e-mail: s.saleem@ee.ucl.ac.uk)

M. Korsah, S. Speller and C.R.M. Grovenor are based in the Department of Materials, Oxford University, Oxford, UK.

S.Saleem acknowledges the financial support from national University of Sciences and Technology (NUST), Pakistan for funding his PhD studies and also the Pakistan Navy for permission to undergo PhD studies. 
ion beam current.

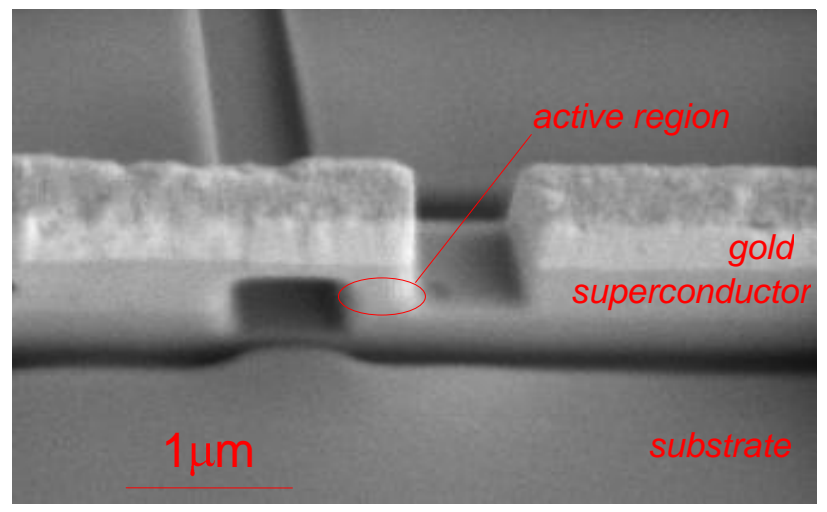

Fig. 1. SEM image of a fabricated IJJ array.

An SEM image of a fabricated junction array is shown in Fig. 1. The top layer, with bright contrast in Fig. 1, is gold. Underneath the gold is the superconducting TBCCO film and the bottom layer is the substrate. The bias current flows through the superconducting film on the left side and is forced to go down through the stack of junctions (marked "active region") and finally leaves the device on the right side. The height of the active region determines the total number of Josephson junctions in the stack. In the array reported here the stack height is $150 \mathrm{~nm}$ and the cross-sectional area of each junction in the array is $1 \mu \mathrm{m}^{2}$. This area was chosen in order to reduce the Ohmic power dissipation in the array by comparison with arrays of larger area.

After fabricating the junctions, the substrate is mounted on the copper block of a chip carrier. The chip carrier is mounted at the end of a dip probe and liquid helium is used to cool the sample down to $4.2 \mathrm{~K}$. We use a custom-built swept DC current source (with output impedance in the $100 \mathrm{k} \Omega$ range) to current-bias the junction stack. The voltage is measured in a four-terminal configuration. In order to irradiate the junctions we use an HP 8671B synthesized CW generator which can provide a power output up to $13 \mathrm{dBm}$. The microwaves are guided to the sample space along a mini-coaxial waveguide. The waveguide is terminated in a dipole antenna. Since there are many parasitic resonances in the sample space, all measurements reported here are performed at a fixed microwave frequency of $6.37 \mathrm{GHz}$. Note that this frequency is about two orders of magnitude lower than the plasma frequency of our junctions. We therefore do not expect to observe any resonant phenomena associated with plasma oscillations in the junction array.

\section{RESULTS AND DISCUSSION}

The dependence of the DC current-voltage characteristics of the junction stack upon the RF power level at a frequency of 6.37 $\mathrm{GHz}$ is shown in Fig. 2. At zero microwave power the current-voltage characteristics are multi-branched and hysteretic. The supercurrent branch is at zero voltage. Once the current bias exceeds the switching current of the supercurrent branch (here 7.6 $\mu \mathrm{A}$ ), it is possible to sweep out $N$ quasiparticle branches where $N$ is the number of IJJs in the stack. ( $N$ is approximately 100 for junction array reported here.) For clarity only the first and second quasiparticle branches are shown in Fig. 2.

For non-zero microwave power, two main changes in the current-voltage characteristics may be observed. The switching currents of the supercurrent branch and (to a lesser extent, as described below) the quasiparticle branches are suppressed. Also an additional branch appears at voltages lower than $5 \mathrm{mV}$. The dependence of the voltage at the top of this low-voltage branch (as illustrated by the arrows in Fig. 2(b)) upon the microwave current is shown in Fig. 3. At RF currents higher than a threshold of 0.04 units the dependence is linear, confirming that the dissipation mechanism on this branch is microwave-induced Josephson vortex-flow [7]-[10].

It is striking that this microwave-induced vortex-flow branch only appears close to the supercurrent branch; when a single intrinsic junction is in the voltage state the vortex-flow branch is totally suppressed. There are two possible interpretations of this observation: (i) when one junction is in the voltage state the dissipation due to quasiparticle tunnelling suppresses the flow of Josephson vortices; or (ii) when one junction is in the voltage state there are no Josephson vortices in the array. 
IEEE Trans. Appl. Supercond. (2009) 19734

http://dx.doi.org/10.1109/TASC.2009.2019226

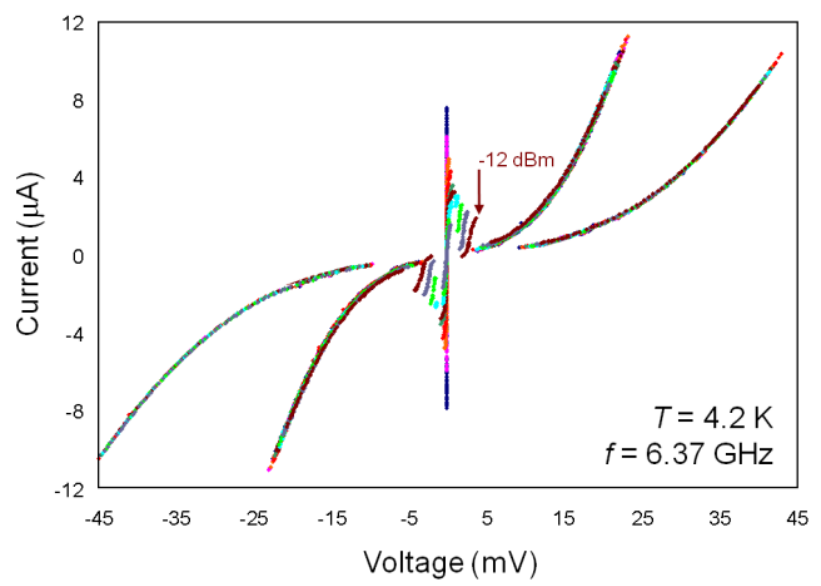

(a)

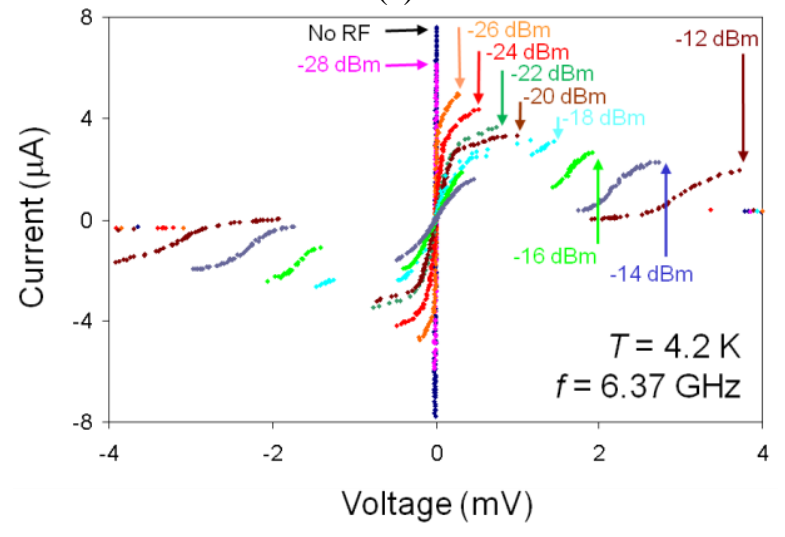

(b)

Fig. 2. (a) $4.2 \mathrm{~K}$ current-voltage characteristics of the junction array as the microwave irradiation power is varied from zero to $-12 \mathrm{dBm}$. Only the supercurrent branch and the first two quasiparticle branches are shown. The arrow indicates the vortex-flow branch when the microwave power is $-12 \mathrm{dBm}$. On the quasiparticle branches the current-voltage characteristics for all microwave powers lie on top of each other. (b) Low voltage region of the characteristics. The arrows indicate the maximum voltage on the vortex-flow branch. Power levels are referred to the output of the microwave source.

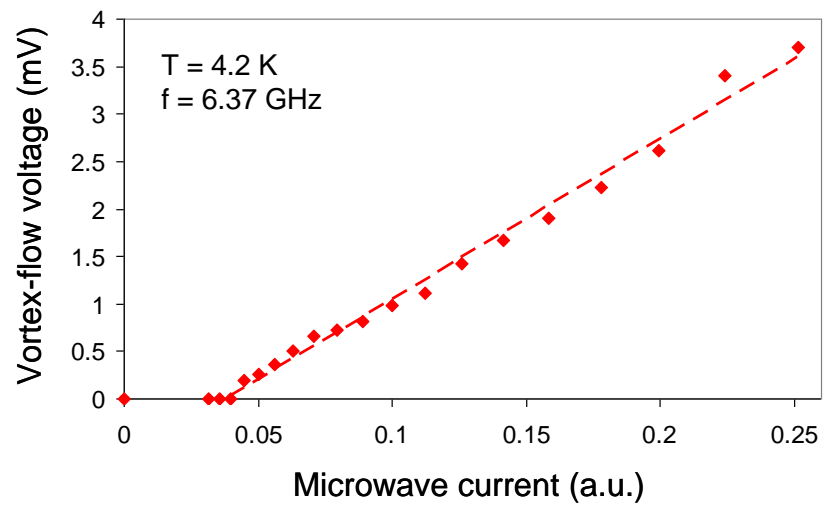

Fig. 3 The maximum voltage of the vortex flow branch (as indicated by the arrow in Fig. 2 (b)) as a function of the RF current in arbitrary units measured at 4.2 $\mathrm{K}$. The line is a linear fit to the data above 0.04 microwave current units.

In order to address this issue we plot the dependence of the first three switching currents upon the applied microwave current in Fig. 4. Here "branch $n$ " refers to switching from the $n$th to the $(n+1)$ th quasiparticle branch for $n \geq 1$. "Branch zero" refers to switching from the supercurrent branch at RF currents up to 0.04 units and switching from the microwave-induced vortex-flow branch at higher RF currents. We observe that the switching current on the zeroth branch is rapidly suppressed by the RF current, whereas that of the subsequent branches is only marginally suppressed. (Scatter in the measurements on branches 1 and 2 is due to thermal fluctuations.) We therefore conclude that the microwave field couples much less strongly to the IJJ array when a single intrinsic junction is in the voltage state than when all the junctions are in the superconducting state. This accounts for our observation that the microwave-induced Josephson vortex-flow branch can only be observed when all the junctions are in the 
superconducting state; since the microwave field couples poorly when a single junction is in the voltage state, no Josephson vortices are inserted in the junction stack.

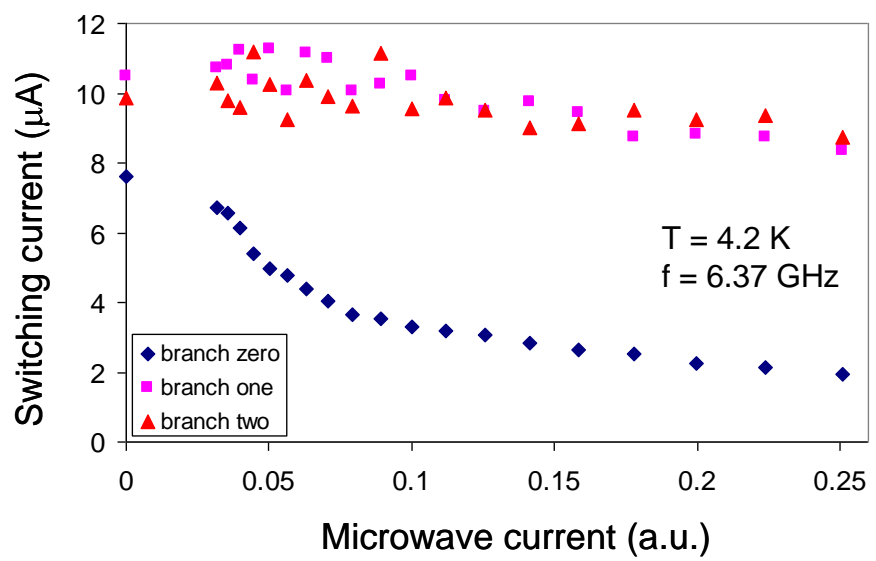

Fig. 4 Dependence of the switching current of the zeroth branch (see text for definition) and the first and second quasiparticle branches on the RF current at $4.2 \mathrm{~K}$.

In our previous work [11] we have seen that thermally activated phase diffusion occurs only on the supercurrent branch and not on the quasiparticle branches. Phase diffusion can only be observed in hysteretic Josephson junctions when the junction resistance is larger than the RF impedance of the environment which is of order $100 \Omega$ [12]. This suggests that the environmental impedance may also be playing a role in determining how well the microwave field couples to our junction array. A simple circuit model to account for this is shown in Fig. 5. Here we model the microwave irradiation by using an RF current source. When all the junctions are in the superconducting state the RF impedance of the junction array is low. (The inductive reactance of a Josephson junction with critical current of order $10 \mu \mathrm{A}$ at $10 \mathrm{GHz}$ is of order $1 \Omega$.) Hence the RF current flows through the junction array. The AC resistance of a single junction in the voltage state (determined from the slope of the current-voltage characteristics, Fig. 2) is between $1 \mathrm{k} \Omega$ and $10 \mathrm{k} \Omega$. With one junction in the voltage state therefore the junction is effectively shorted by the environmental impedance and very little RF current couples to the junction array.

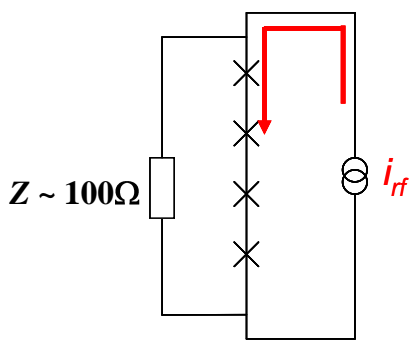

Branch zero

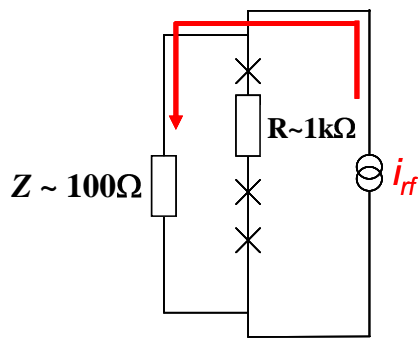

Branch one

(a)

(b)

Fig. 5 RF equivalent circuit model to show the difference in RF coupling to an array of Josephson junctions. $\mathrm{Z}$ represents the environmental impedance. In (a) all the junctions are in the zero voltage state and the RF current flows through the array. In (b) one junction is in the voltage state and is modelled by a resistor of value of order $1 \mathrm{k} \Omega$. Here the majority of the RF current flows through the environmental impedance.

\section{CONCLUSION}

How RF fields are distributed within the stack of intrinsic junctions is important in the development of high-power narrowlinewidth tuneable THz emitters. Bae et al. [13] showed that below some cut-off frequency (in their case around $80 \mathrm{GHz}$ ) the RF fields were uniformly distributed in the junction stack, whereas above this cut-off frequency a standing wave was set up between the top and the bottom of the stack. In our experiments we irradiate at a much lower frequency than in [13] so that we can expect uniform irradiation across the stack provided the radiation couples to the junction at all. As we have shown in our experiments such coupling only occurs when all the junctions are in the superconducting state. The difference between our data and those of Bae et al. arises from the much smaller junction area in our experiments - in the larger junctions (of area $18 \mu \mathrm{m}^{2}$ ) in [13] the much lower junction resistance means that the environmental impedance plays no role.

The problem of coupling microwaves to small area intrinsic junctions is one (of many) which must be addressed if intrinsic 
junctions are to be developed as qubits. Further work is therefore under way to develop compact resistors which can be placed close to the junction stack so as to isolate it from the environment [14].

\section{REFERENCES}

[1] R. Kleiner, F. Steinmeyer, G. Kunkel, P. Muller, “Intrinsic Josephson effects in $\mathrm{Bi}_{2} \mathrm{Sr}_{2} \mathrm{CaCu}_{2} \mathrm{O}_{8}$ single crystals,” Phys. Rev. Lett, vol. 68, p. 2394 , 1992.

[2] L. Ozyuzer et al, "Emission of Coherent THz Radiation from Superconductors," Science, vol. 318, p. 1291, 2007

[3] J. Faist et al, "Quantum Cascade Laser," Science, vol. 264, p. 553, 1994

[4] John M. Martinis et al., "Decoherence in Josephson Qubits from Dielectric Loss," Phys. Rev. Lett, vol. 95, art. no. 210503, 2005.

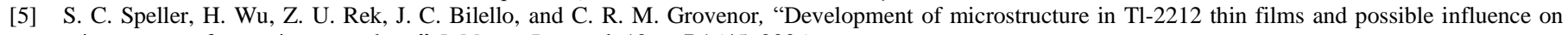
microwave surface resistance values," J. Mater. Res., vol. 12, p. R1645, 2006.

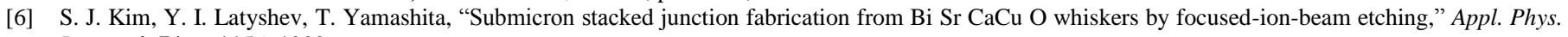
Lett., vol. 74, p. 1156, 1999.

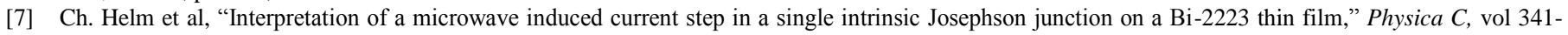
348 , p. 2645, 2000

[8] F. L. Barkov, M. V. Fistul, A. V. Ustinov, "Microwave-induced flow of vortices in long Josephson junctions," Phys. Rev. B, vol. 70 , art. no. 134515, 2004.

[9] K. Hirata, S. Ooi, T. Mochiku, "Excitation of Josephson plasma by Josephson vortex flow," Physica C, vol. 362, p. 114, 2001

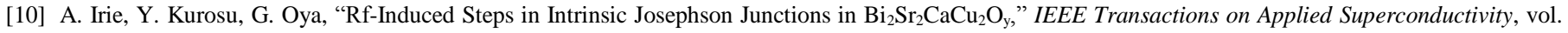
13, no. 2 , p. 908,2003

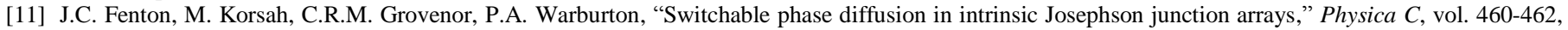
p. 1470,2007

[12] M. Tinkham, Introduction to superconductivity, 2nd ed. McGraw-Hill, New York, 1996

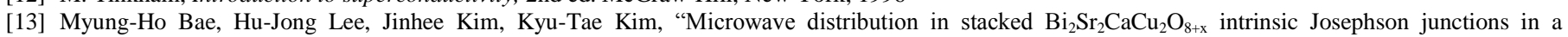
transmission-line geometry," Appl. Phys. Lett, vol. 83, p. 2187, 2003.

[14] L. S. Kuzmin, D. B. Haviland, "Observation of the Bloch oscillations in an ultrasmall Josephson junction," Phys. Rev. Lett., vol. 67, p. 2890, 1991 